\title{
Beam optics of gain-guided soft-x-ray lasers in cylindrical plasmas
}

\author{
Juan L. A. Chilla and Jorge J. Rocca \\ Department of Electrical Engineering, Colorado State University, Fort Collins, Colorado 80523
}

Received September 21, 1995; revised manuscript received June 3, 1996

\begin{abstract}
We analyze soft-x-ray beam propagation and amplification in gain-guided amplifiers of cylindrical geometry for arbitrary gain and density profiles. A general relation that must be fulfilled for refraction not to be an impediment to the exponential growth of the intensity is obtained. It is shown that for sufficiently long plasma columns the effective gain, reduced by refraction, is determined solely by the gain and the curvature of the density profile at the position of maximum density, even when the location of the gain and the density maxima do not coincide. We analyze the case of amplifiers with gain-length approaching saturation and show that refraction reduces the effective gain-length product at which gain saturation occurs. The theoretical results are used to analyze the output of a capillary discharge soft-x-ray laser. (c) 1996 Optical Society of America.
\end{abstract}

\section{INTRODUCTION}

Soft-x-ray lasers utilize as a gain medium high-density plasmas of small lateral dimensions, in which refraction of the amplified x-ray radiation can result from electrondensity gradients. Until recently, the only successful soft-x-ray amplification experiments were conducted in laser-created plasmas. ${ }^{1}$ In the most common pumping configuration a powerful laser is focused onto a thin foil or a slab target to create the hot dense plasma in which population inversion is created. In Ref. 2, London has studied the beam optics of exploding foil x-ray lasers, which are well described by a model with a onedimensional density gradient, assuming parabolic density profiles.

Recently, large soft-x-ray amplification was demonstrated for the first time in a plasma column created by a discharge. $^{3} \quad$ A fast capillary discharge was utilized to generate amplification in the $J=0-1$ line of neonlike argon at $46.9 \mathrm{~nm}$ by collisional electron excitation. In this excitation scheme a fast current pulse rapidly detaches the plasma column from the capillary walls and compresses it, creating a hot plasma column of small diameter and cylindrical symmetry. ${ }^{4}$ Toward the end of the compression stage, the necessary plasma conditions for soft-x-ray amplification by collisional excitation of neonlike ions are obtained. The analysis of the soft-x-ray beam propagation and amplification in such a plasma column requires one to take into account the twodimensional variation of the plasma parameters. The problem of gain guiding in a plasma of cylindrical geometry was previously considered by Fill. ${ }^{5}$ That analysis is, however, restricted to Gaussian beams and therefore cannot account for non-Gaussian beam profiles that occur for highly refractive plasmas, even in the case of parabolic density profiles.

In this paper we analyze soft-x-ray beam propagation and amplification in a plasma of cylindrical geometry for arbitrary gain and density profiles. The analysis presented here reduces to that of Ref. 2 when restricted to one-dimensional gradients and parabolic profiles. While this work concentrates on gain-guided configurations, for completeness we include a brief discussion of the case in which density profiles lead to index-guided situations. ${ }^{6,7}$ The next section describes the formalism employed in the calculations. In Section 3 we analyze the particular case of parabolic gain and density profiles, which forms the basis for the analysis of the general case of profiles of arbitrary shape presented in Section 4 . The results of numerical integration for several selected gain and density profiles are presented in Section 5, and the case of an amplifier with gain-length approaching saturation is discussed in Section 6. In Section 7 the theoretical results are used to analyze the measured characteristics of a capillary discharge pumped soft-x-ray amplifier.

\section{RAY TRAJECTORIES AND FORMALISM}

In order to compute the output beam profile we need knowledge of the ray trajectories within the plasma. Our starting point is the ray-propagation equation ${ }^{8}$ :

$$
\frac{\mathrm{d}}{\mathrm{d} s}\left(\eta \frac{\mathrm{d} \mathbf{r}}{\mathrm{d} s}\right)=\nabla \eta
$$

where $\mathrm{d} s$ is the differential path length, $\mathbf{r}$ is the ray position vector, and $\eta$ is the position-dependent index of refraction. We will deal with a geometry such as that shown in Fig. 1, with cylindrical symmetry around the $z$ axis, along which the amplified radiation is emitted. The electron density is mostly concentrated in a cylinder of radius $a$ around the $z$ axis, and hence the index of refraction departs significantly from unity only in that region of space. The index of refraction is related ${ }^{9}$ with the electron density $n_{e}$ through

$$
\eta=\sqrt{1-\frac{n_{e}}{n_{c}}}
$$




$$
n_{c}=\frac{\pi m_{e} c^{2}}{e^{2} \lambda^{2}}
$$

where $n_{c}$ is the critical density at the x-ray laser wavelength $\lambda$.

To take advantage of the cylindrical geometry, we can express the position vector as $\mathbf{r}=r \hat{\rho}+z \hat{z}$ in terms of $\hat{z}$ and $\hat{\rho}$, unit vectors parallel and perpendicular to the $z$ axis, respectively. The vector $\hat{\rho}$ forms an angle $\theta$ with the $x$ axis. Because of the cylindrical symmetry, $\nabla \eta$ $=\mathrm{d} \eta / \mathrm{d} r \hat{\rho}$. The gain region, which is also assumed to have cylindrical symmetry, can in general have a profile different from that of the electron density. Rays emitted in directions close to the $z$ direction travel longer distances through the gain region, experiencing more amplification. These rays are thus more intense than rays emitted in other directions. Taking this fact into account, derivatives in the direction of the ray trajectories can be replaced by $\mathrm{d} / \mathrm{d} z$, and a term containing the derivative of $\eta$ with respect to $s$ can be neglected for being a small number multiplied by the scalar product of two almost perpendicular vectors. With these considerations the propagation equation in cylindrical coordinates becomes

$$
\begin{aligned}
\frac{\mathrm{d}^{2} r}{\mathrm{~d} z^{2}}-r\left(\frac{\mathrm{d} \theta}{\mathrm{d} z}\right)^{2} & =\frac{\mathrm{d}}{\mathrm{d} r} \ln (\eta), \\
2 \frac{\mathrm{d} r}{\mathrm{~d} z} \frac{\mathrm{d} \theta}{\mathrm{d} z}+r \frac{\mathrm{d}^{2} \theta}{\mathrm{d} z^{2}} & =0 .
\end{aligned}
$$

The solution of this system of coupled equations for $r$ and $\theta$ gives the trajectory of any ray propagating through the plasma column. As we show in Section 4, only those rays in radial trajectories, following the simpler equation $\mathrm{d}^{2} r / \mathrm{d} z^{2}=\mathrm{d} / \mathrm{d} r \ln (\eta)$, are relevant for the calculation of the output intensity for long plasma columns.

To calculate the far-field beam pattern, we follow the procedure outlined by London; ${ }^{2}$ we integrate the emergent specific intensity $I$ over the surface area of the lasing medium as viewed by the detector. The specific intensity is defined ${ }^{2}$ as the quantity of radiant energy passing a

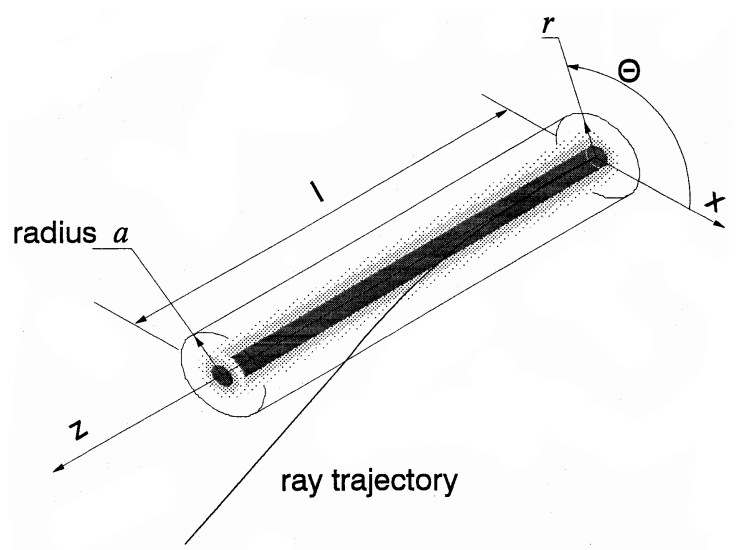

Fig. 1. Schematic representation of the cylindrical plasmacolumn geometry under consideration. A typical ray-trajectory bending caused by refraction is shown and variables used in the text are defined. unit area in a specified direction, per unit intervals, in time, solid angle, and frequency. For a particular ray the intensity depends only on its gain-length product $G$ $=\int g \mathrm{~d} s$ and can be computed as

$$
I(G)=S\left(e^{G}-1\right)
$$

with

$$
\begin{aligned}
& g=c^{2} A_{21} /\left(8 \pi \nu^{2}\right) n_{u}\left(1-h_{u} n_{l} / h_{l} n_{u}\right) \psi(\nu), \\
& S=\left(2 h \nu^{3} / c^{2}\right)\left(1-h_{u} n_{l} / h_{l} n_{u}\right)^{-1},
\end{aligned}
$$

where $g$ and $S$ are, respectively, the gain and the source functions as computed for the relevant line. In Eqs. (5), $A_{21}$ is Einstein's $A$ coefficient for the transition, $\nu$ is its frequency, and $h$ and $n$ are the statistical weights and populations of the upper and the lower laser levels as indicated by the subscripts. These formulas assume that there is no incident radiation at $z=0$ and take into account the contribution of spontaneous emission to the intensity along the entire length of the gain medium. As in Ref. 2 both the spontaneous and the stimulated emission have the same line profile function $\psi(\nu)$ and spatial dependence.

Because of the cylindrical symmetry, we only need to compute the angular dependence of the far-field emission pattern for rays emerging from the plasma in directions parallel to the $x-z$ plane. With the subscript 2 we indicate the coordinates at the exit plane of the plasma; thus $\left(r_{2}, \theta_{2}, l\right)$ are the cylindrical coordinates of the point where a ray forming an angle $\phi_{2}$ with the $z$ axis intersects the exit plane. The beam pattern is computed from the following integral over the exit plane:

$$
F\left(\phi_{2}\right)=\iint I\left(r_{2}, \theta_{2}\right) r_{2} \cos \left(\phi_{2}\right) \mathrm{d} r_{2} \mathrm{~d} \theta_{2} .
$$

With knowledge of the density profile and the position and the slope of the ray at $z=l$, namely, $r=r_{2}, \theta$ $=\theta_{2}, \mathrm{~d} r / \mathrm{d} z=\phi_{2} \cos \theta_{2}, \quad$ and $\quad \mathrm{d} \theta / \mathrm{d} z=-\phi_{2} \sin \theta_{2} /$ $r_{2}$, Eqs. (3) can be solved to obtain the ray trajectories. The gain profile can be used to compute the gain-length $G$ and finally the integral in Eq. (6).

\section{PARABOLIC PROFILES}

In this section we analyze the case of a cylindrical plasma with parabolic density and gain profiles having the same radius $a$, extending to cylindrical geometry the unidimensional analysis by London. ${ }^{2}$ Parabolic profiles are a good approximation to a variety of smooth profiles likely to be found in some real amplifier systems while still being simple enough to allow the existence of analytical solutions for most of the equations found in the calculation of the beam profiles:

$$
\begin{aligned}
& n(r)=n_{e}\left[1-(r / a)^{2}\right], \\
& g(r)=g_{0}\left[1-(r / a)^{2}\right] .
\end{aligned}
$$

For a parabolic density profile the propagation equation is separable and easier to solve in Cartesian coordinates. With the refraction length $L_{r}=a \sqrt{n_{c} / n_{e}}$, the typical distance traveled by the ray before exiting the laser medium, ${ }^{2}$ the trajectory can be expressed as 


$$
\begin{aligned}
& x=A_{x} \exp \left(z / L_{r}\right)+B_{x} \exp \left(-z / L_{r}\right), \\
& y=A_{y} \exp \left(z / L_{r}\right)+B_{y} \exp \left(-z / L_{r}\right),
\end{aligned}
$$

where the integration constants $A_{x, y}$ and $B_{x, y}$ are obtained from the position and the slope of the ray when it exits the plasma:

$$
\begin{aligned}
& A_{x}=\frac{1}{2}\left(r_{2} \cos \theta_{2}+\phi_{2} L_{r}\right) \exp \left(-z_{2} / L_{r}\right), \\
& A_{y}=\frac{1}{2}\left(r_{2} \sin \theta_{2}\right) \exp \left(-z_{2} / L_{r}\right), \\
& B_{x}=\frac{1}{2}\left(r_{2} \cos \theta_{2}-\phi_{2} L_{r}\right) \exp \left(z_{2} / L_{r}\right), \\
& B_{y}=\frac{1}{2}\left(r_{2} \sin \theta_{2}\right) \exp \left(z_{2} / L_{r}\right) .
\end{aligned}
$$

The gain-length product $G$ for a given ray is obtained after an analytical integration as a function of $z_{1}$ and $z_{2}$, the initial and exit positions of the ray, and of the constants $A_{x, y}$ and $B_{x, y}$ :

$$
\begin{aligned}
G= & \frac{g_{0} L_{r}}{2 a^{2}}\left\{2 \frac{\left(z_{2}-z_{1}\right)}{L_{r}}\left[a^{2}-2\left(A_{x} B_{x}+A_{y} B_{y}\right)\right]\right. \\
& -\left(A_{x}^{2}+A_{y}{ }^{2}\right)\left[\exp \left(2 z_{2} / L_{r}\right)-\exp \left(2 z_{1} / L_{r}\right)\right] \\
& +\left(B_{x}^{2}+B_{y}{ }^{2}\right) \times\left[\exp \left(-2 z_{2} / L_{r}\right)\right. \\
& \left.\left.-\exp \left(-2 z_{1} / L_{r}\right)\right]\right\} .
\end{aligned}
$$

Finally, for a particular configuration of the plasma column, the dimensions and the gain values should be replaced and the integral in Eq. (6) should be performed numerically in order to be able to account for all the possible trajectories. As an illustrative example, we will consider a plasma column of radius $a=100 \mu \mathrm{m}$, with a central gain $g_{0}$ of $2 \mathrm{~cm}^{-1}$, and having an electron density that produces a refraction length $L_{r}$ of $2.5 \mathrm{~cm}$. In our discussion we will use the fact shown by London ${ }^{2}$ that, for parabolic profiles, most of the results of the analysis depend on ratios of the quantities to the characteristic values $\phi_{r}$ $=\left(n_{e} / n_{c}\right)^{1 / 2}$ and $L_{r}$. Figure 2 shows the result of the numerical integration for three different laser-medium lengths. Figure 2(a) shows the far-field pattern that is obtained when the length of the gain medium is $l=L_{r}$; such a short laser medium behaves not very differently from a purely spontaneous emitter, and the effect of the gain is seen as a higher intensity in the direction of the $z$ axis. The combined effect of refraction and gain guiding is already noticeable in Fig. 2(b), which shows the farfield pattern for a longer laser medium $l=3 L_{r}$. The output radiation can be seen to have two distinct components, the most important of them centered on axis having a Gaussian shape, the other a small peak centered at the refraction angle $\phi_{r}$. The on-axis feature corresponds to the case studied in Ref. 5 , where both the real and the imaginary parts of the index of refraction have a quadratic dependence in the radial coordinate and hence define a waveguide. As the real part of the index is minimum on axis, refraction causes a leakage of radiation to the sides, while the imaginary part (gain) provides the confinement action. For the set of parameters chosen in this example the beam pattern is already very similar to that of the mode of the waveguide. The off-axis feature is a consequence of the fact that the index of refraction stops growing at $r=a$, the outer limit of the plasma column. As the index is constant outside the plasma, those rays that emerge from the sides of the plasma column keep their exit direction, which for relatively long plasma columns forms an angle $\phi_{r}$ with the axis.

Figure 2(c) shows the beam profile for $l=6 L_{r}$, with significant changes in respect to Fig. 2(b). The flux of the on-axis Gaussian feature continues to grow, keeping its width as defined by the waveguide. The flux of the refracted component also grows but gets narrower with increasing plasma-column length. By taking advantage of the relative simplicity of the resulting expressions with parabolic profiles, we can obtain approximate formulas for the angular distribution of the flux of the on-axis and off-axis components ( $F_{1}$ and $F_{2}$, respectively):

$$
\begin{aligned}
F_{1}\left(\phi_{2}\right)= & \frac{8 S a^{2} \pi}{g_{0} L_{r}} \exp \left[\left(g_{0}-2 / L_{r}\right) l-g_{0} L_{r}\left(\phi_{2} / \phi_{r}\right)^{2} / 2\right] \\
F_{2}\left(\phi_{2}\right)= & \sqrt{\frac{8 \pi}{g_{0} L_{r}}} \frac{S a^{2}}{\left(g_{0} L_{r}-1\right)} \exp \left[\left(g_{0}-1 / L_{r}\right) l\right. \\
& \left.-g_{0} L_{r} / 2-\frac{\left(\phi_{2}-\phi_{r}\right)^{2}}{2 \sigma^{2}}\right]
\end{aligned}
$$
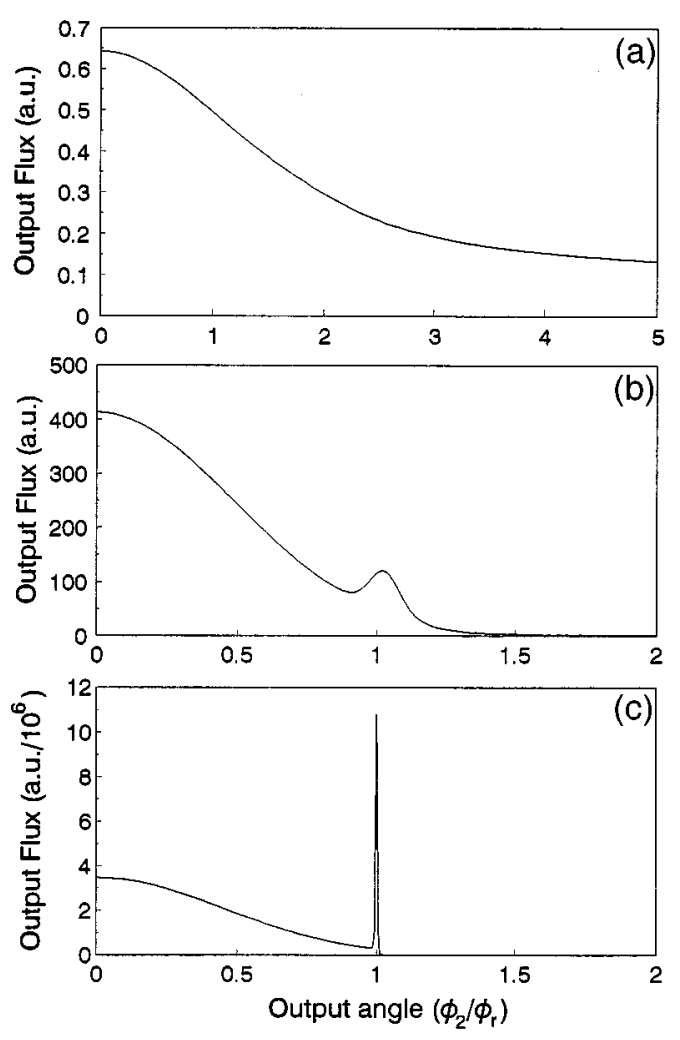

Fig. 2. Output beam profile for parabolic index and gain profiles computed for different plasma-column lengths. For these calculations, $g_{0} L_{r}=5$, and the plasma-column length $l$ was set to (a) $L_{r}$, (b) $3 L_{r}$, and (c) $6 L_{r}$. The units of flux are arbitrary but consistent for all plots. 
with

$$
\sigma=\frac{4 \phi_{r}^{2}}{g_{0} L_{r}} \exp \left(-2 l / L_{r}\right)
$$

Both components display an exponential dependence on the plasma-column length. While for the case of $F_{1}$ the flux increases with an effective gain equal to $g_{0}$ $-2 / L_{r}$, for $F_{2}$ the gain is $g_{0}-1 / L_{r}$. However, as the angular width $\sigma$ of the peak decreases exponentially with plasma-column length, the gain for the off-axis x-ray laser power (after angular integration) is $g_{0}-2 / L_{r}$, the same as for the on-axis component. This shows that refraction introduces a loss term $1 / L_{r}$ for each dimension, generalizing for a cylindrical (two-dimensional) geometry, the result obtained by London. ${ }^{2}$ From the above expression for the effective gain it follows that the length dependence of the laser intensity corresponds to one of two cases determined by whether the refraction gain-length $G_{r}=g_{0} L_{r}$ is smaller or larger than 2 (instead of smaller or larger than 1 , as found in the one-dimensional case). If the gain is small or the density gradients are too steep $\left(G_{r}<2\right)$, gain cannot overcome the refraction losses and amplification is limited by refraction. If the gain is sufficiently high $\left(G_{r}>2\right)$, exponential growth of the intensity continues until saturation of the gain medium is achieved.

By examination of Eqs. (12) we find exponential factors that determine the length dependence of the flux multiplied by constants that account for the space integral involved in the computation. The exponential dependence corresponds to that of the intensity of a particular set of ray trajectories $\left(B_{x, y}=0\right)$ that stay within the plasma column for its entire length. This means that for a sufficiently long plasma column both the length dependence of the flux and the spatial profile of the beam are solely determined by the amplification of the particular set of rays that stay within the plasma column for its entire length. All other rays will experience less amplification, and its effect will be negligible after a certain plasmacolumn length. We will use this fact when analyzing arbitrary profiles in the next section. The analysis of the general case is motivated by the fact that a parabolic density profile is not likely to be a good approximation for cases such as that of a plasma column created by the strong compression shock of a discharge. In that case, more highly peaked profiles can arise.

\section{ARBITRARY GAIN AND DENSITY PROFILES}

\section{A. Optimal Rays}

For a given density profile and output angle $\phi_{2}$ there is an infinite number of possible ray trajectories, all of them contributing to the output flux. However, because of the exponential dependence of the intensity on the gainlength product, the output flux can be approximately calculated by taking into account only those ray trajectories that stay within the plasma column for its entire length. For short plasma columns, refraction does not have an important effect, and almost all rays initially emitted within the solid angle subtended by the plasma column fall within this category. With increasing plasmacolumn length the subset of rays not being bent out by re- fraction becomes smaller, and in the limit of a very long column it reduces to a particular set of rays that characterizes completely the output beam profile and the length dependence of the intensity of gain-guided lasers. These rays have a precise mathematical definition and can be identified from the differential Eq. (3) without knowledge of the precise shape of $\eta(r)$. The mathematical form of these equations is similar to that of the motion of a particle in two dimensions ( $r$ and $\theta$ ) in a central potential $[-\ln (\eta)]$. In a way analogous to the study of kinematics we can extract constants of motion, quantities that are constant along the trajectory of any ray:

$$
\begin{aligned}
& C_{1}=\left(\frac{\mathrm{d} r}{\mathrm{~d} z}\right)^{2}+r^{2}\left(\frac{\mathrm{d} \theta}{\mathrm{d} z}\right)^{2}-2 \ln (\eta), \\
& C_{2}=r^{2} \frac{\mathrm{d} \theta}{\mathrm{d} z} .
\end{aligned}
$$

The constants $C_{1}$ and $C_{2}$ are analogous to the kinematic quantities total energy and angular momentum, respectively. It follows from the second equation in Eqs. (13) that the rays that intersect the $z$ axis are restricted to radial trajectories and, conversely, the rays that in some point have a nonzero $\mathrm{d} \theta / \mathrm{d} z$ will not intersect the $z$ axis. Because of the second term in the first equation in Eq. (13), analogous to a centrifugal acceleration, corkscrewing rays $(\mathrm{d} \theta / \mathrm{d} z \neq 0)$ always stay in regions with high gain for distances shorter than those of radial rays. By considering only radial rays $(\mathrm{d} \theta / \mathrm{d} z=0)$ and solving for $\mathrm{d} r / \mathrm{d} z$ the first equation in Eqs. (13), we obtain

$$
\frac{\mathrm{d} r}{\mathrm{~d} z}=\sqrt{2 \ln \left(\frac{\eta}{\eta_{0}}\right)+C_{1}},
$$

where $\eta_{0}$ is the value of the index of refraction on the $z$ axis. By appropriately choosing those rays for which the value of the constant $C_{1}=2 \ln \left[\eta_{0} / \eta\left(r_{m}\right)\right]$, we obtain a set of ray trajectories that we call optimal rays. Each optimal ray is parallel to the axis when the ray is at $r_{m}$, the radial position corresponding to the maximum electron density and hence the minimum index of refraction. A characteristic feature of optimal rays is that, except for a short distance close to the end of the plasma column, their trajectories stay very close to the position of maximum density. Some optimal rays stay within the gain region for the entire length of the plasma in the $z$ direction for any length of the plasma column and are thus the ones experiencing the largest amplification. When the maximum electron density is located on axis [Fig. 3(a), $r_{m}$ $=0]$, optimal rays are the only ones staying within the gain medium the entire length of the gain medium. When the maximum electron density is in a ring [Fig. $3(b)]$, an index waveguide arises, ${ }^{6,7}$ and other rays can also be found that have this property.

For long plasmas, besides optimal rays that emerge from the plasma column through the end face, the next most important contribution to the far-field emission pattern is from those optimal rays that emerge from the plasma through the side near the end of the plasma column. The end-face radiation will be centered on axis, and its angular distribution will depend on the details of the electron-density and gain distributions. The refracted radiation will not alter its direction after emerg- 
ing from the plasma column; consequently it will be concentrated at the angle, $\phi_{r}$, that optimal rays have at the exit position, $r=a$. By substituting Eq. (2) into Eq. (14) and neglecting higher-order terms in $n_{e} / n_{c}$, we obtain an expression for $\phi_{r}$ that depends only on the maximum electron density $n_{e}$ :

$$
\phi_{r}=\sqrt{\frac{n_{e}}{n_{c}}} .
$$

This result was previously obtained by London ${ }^{2}$ for a unidimensional parabolic density profile, but as shown above is valid in general. Although in all the situations considered in this paper the region outside the plasma column $(r>a)$ is transparent, profiles displaying absorption in this region are possible; for such profiles the off-axis feature would be less important. The length dependence of the output intensity is obtained next also by taking advantage of the properties of optimal rays.

\section{B. Long Plasma Columns}

As often refraction effects are difficult to avoid and intrinsic to the process of generation of the gain medium for x-ray lasers, an important question that must be addressed is whether refraction is strong enough to stop the amplification of spontaneous emission before gain saturation is achieved. This is highly dependent on the shape of the gain and the density profiles, but, as we will show in this section, it is possible to generalize the refraction gain-length $G_{r}$ defined in connection with parabolic profiles $^{2}$ to determine a general relation that must be fulfilled to maintain exponential growth until the onset of gain saturation.

Let us first consider the situation schematically depicted in Fig. 3(a), in which the density is assumed to be maximum on the $z$ axis. As previously discussed, the output flux can be approximately calculated by considering only the rays that stay within the plasma column for its entire length. For sufficiently long plasma columns, only optimal rays fulfill that condition. As a consequence of this, the length dependence of the flux in the limit of
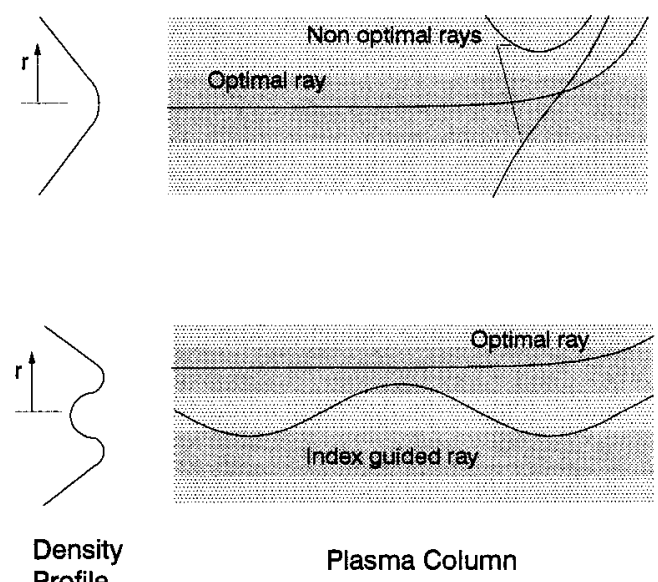

Fig. 3. Schematic representation of ray trajectories in a cylindrical plasma column for arbitrary density profiles with maximum (a) on axis and (b) off axis. As discussed in the text, for long plasma columns, only optimal and index-guided rays are relevant for the calculation of the output beam profile. long plasma columns will be determined solely by the gain-length product of optimal rays. Even if the maximum gain is not located on axis and optimal rays spend most of their time in regions with low gain, for long plasma columns they eventually achieve larger gainlength products than rays that go through high-gain regions but are bent out of the plasma column in relatively short distances. For any physically realizable density profile the region closest to the maximum can be well approximated locally by a parabola, and thus formulas similar to Eqs. (12) can be employed to compute the output flux:

$$
F\left(\phi_{2}\right)=\mathscr{C} \mathscr{F}\left(\phi_{2}\right) \exp \left[\left(1-2 / G_{r}\right) g_{0} l\right] .
$$

In this equation, $\mathscr{C}$ represents a constant that originates in the spatial integration, and $\mathscr{F}\left(\phi_{2}\right)$ is the angular dependence of the far-field profile, none of which can be computed without knowledge of the shape of the gain and the density profiles. Equation (16) gives, nevertheless, the length dependence of the flux $\left[F\left(\phi_{2}\right)\right]$, which is determined by the gain and the curvature of the index profile at the position of maximum density and is characterized by the parameter

$$
G_{r}=\frac{g\left(r_{m}\right)}{\sqrt{\partial^{2} \eta / \partial r^{2}}} \approx g\left(r_{m}\right) \sqrt{-\frac{2 n_{c}}{\partial^{2} n_{e} / \partial r^{2}}} .
$$

This new general definition of $G_{r}$ includes that defined by London $^{2}$ and discussed in Section 3 as a particular case. In general, for a cylindrical geometry, when $G_{r}>2$, the flux grows exponentially with an effective gain that is reduced by refraction from the on-axis value $g_{0}$ by a factor $1-2 / G_{r}$. On the other hand, when $G_{r}<2$, refraction stops the exponential growth.

When the maximum density is not located on axis, as schematically depicted in Fig. 3(b), the situation is slightly more complicated because optimal rays are not the only ones staying within the plasma column for its entire length. In this configuration the contribution of optimal rays to the total output flux can be computed analogously to the calculations leading to Eq. (16), with some differences caused by the unidimensional nature of the density maximum. As the maximum density is off axis, there is a region of lower index of refraction surrounding the $z$ axis, effectively creating an index waveguide. The mode properties of such a plasma waveguide and its application to soft-x-ray amplification have been studied in Refs. 6 and 7. The particular distribution of rays that constitutes a mode of the waveguide stays indefinitely within the plasma column, experiencing a modal gain $g_{w}$ that can be computed as the convolution of the gain and the mode profiles. The total output flux in the limit of a long plasma column can be expressed as the sum of both contributions:

$$
\begin{aligned}
F\left(\phi_{2}\right)= & \mathscr{C}_{g} \mathscr{F}_{g}\left(\phi_{2}\right) \exp \left[\left(1-1 / G_{r}\right) g\left(r_{m}\right) l\right] \\
& +\mathscr{C}_{w} \mathscr{F}_{w}\left(\phi_{2}\right) \exp \left(g_{w} l\right) .
\end{aligned}
$$

The first term in Eq. (18) is the contribution of the optimal rays (gain guided), and the second term is that of the index-guided rays. $\mathscr{C}$ and $\mathscr{F}$ are, respectively, the spatial integration factor and the angular distribution for 
the contribution of each type of ray. The exponential growth of the gain-guided contribution is reduced by refraction from the gain at the position of maximum density $\left[g\left(r_{m}\right)\right]$ by a factor $1-1 / G_{r}$ caused by the onedimensional density gradient around the position of maximum density. For the same reason, $G_{r}$ should in this case be greater than 1 for refraction not to stop the exponential growth of the intensity. The index-guided contribution, on the other hand, grows exponentially with the modal gain $g_{w}$ and cannot be stopped by refraction. Depending on the gain and the density profiles, the contribution with the highest gain will dominate the far-field pattern for long plasma columns.

\section{Short Plasma Columns}

As previously stated, expressions (16) and (18) are valid for long plasma columns when the most important contribution to the output flux is that of optimal rays or indexguided rays. For short plasma columns it is possible to find rays that stay in regions with high gain and thus cause the flux to grow exponentially with the maximum gain, even for configurations with $G_{r}<2$. By increasing the length of the plasma column, these ray trajectories become less numerous and eventually disappear, and the value of the gain gradually decreases to that of the effective gain. The transition between these two regimes is smooth and depends on the gain and the density profiles, but with suitable approximations it is possible to estimate the plasma-column length at which optimal rays become dominant. That length is determined by the maximum distance that nonoptimal rays are able to stay in the neighborhood of the position of maximum gain before being bent out by the effect of refraction. For configurations in which the gain is not high enough to overcome the refraction losses the transition length gives an estimate of the maximum length for which amplification is obtained.

Let us assume that the gain is significant in a region of radial width $\Delta r$ around the position of maximum gain. In this region we compute the average gradient of the index of refraction $\overline{\mathrm{d} \eta / \mathrm{d} r}$. As the density gradients are solely responsible for the rays bending out, a crude estimate of the transition length can be obtained by the simplification of assuming a constant gradient of the index of refraction in the region surrounding the position of maximum gain. It follows from Eqs. (3) that radial rays are the ones staying longer in the plasma column, and if restricted to radial rays, Eqs. (3) can be solved analytically, resulting in parabolic trajectories $\left(r_{0}\right.$ and $z_{0}$ are integration constants):

$$
r(z)=r_{0}+\frac{\overline{\mathrm{d} \eta / \mathrm{d} r}}{2}\left(z-z_{0}\right)^{2}
$$

These rays stay within the region $\Delta r$ around the position of maximum gain for a distance that depends on the output angle. Rays that are emitted on axis stay within the gain region for a distance around $(2 \Delta r / \overline{\mathrm{d} \eta / \mathrm{d} r})^{1 / 2}$. Rays emitted at $\phi_{2}=\overline{\mathrm{d} \eta / \mathrm{d} r} \Delta r$ are the ones staying the longest distance within the gain region, $2(2 \Delta r / \overline{\mathrm{d} \eta / \mathrm{d} r})^{1 / 2}$, two times longer than rays emitted on axis. For rays emitted at larger angles the distance decreases within increasing emission angle. By applying the above analysis to the case of a plasma column of radius $a$ and maximum electron density $n_{e}$ having smooth profiles with both the density and the gain maxima located on axis, we can expect the flux to reach its steadystate on-axis exponential growth after a length given approximately by $a\left(2 n_{e} / n_{c}\right)^{1 / 2}$. For the particular case of a parabolic density profile this expression indicates that the on-axis steady-state exponential growth is reached at a plasma column of $\sqrt{2} L_{r}$.

It should be noticed that a highly peaked density profile does not necessarily result in low amplification. In that case the large curvature of the density profile around the maximum can lead to high refraction and to a value of $G_{r}<2$. However, if the gain is high enough, the intensity can grow several orders of magnitude before its rapid increase is stopped by refraction. As discussed above, the growth continues for longer plasma columns for rays that are emitted off axis. This configuration produces a beam profile characterized by dominant side lobes that continue to grow for plasma columns of a length up to approximately $2(2 \Delta r / \overline{\mathrm{d} \eta / \mathrm{d} r})^{1 / 2}$.

\section{EXAMPLES}

In this section we present the results of numerical computations of the output flux for a variety of particular gain and density profiles. Although not responding to any particular experiment, the example profiles were chosen for their value in highlighting the features discussed in the previous section. The calculation of the output flux requires the solution of a second-order, two-variables differential equation, and two consecutive integrations on the solution, one involving one variable, and the other two variables. Performing this full procedure numerically requires considerable computation time, which can be reduced if one or more steps can be performed analytically. This consideration dictated the election of some of the example profiles in this section. The two-dimensional integration over the output plane was in all cases performed numerically. This is a particularly difficult integral to perform by numerical methods, as the integrand varies over several orders of magnitude and is highly peaked around a certain position. The integration procedure adopted is a recursive Gauss-Legendre quadrature ${ }^{10}$ with the formulas for five and six points. In order to provide accurate results, we had to adequately partition the integration region. When performed numerically, the integral giving the gain-length $G$ was computed with a 15point Gauss-Legendre quadrature, and the differential equation was solved by a fourth-order Runge-Kutta ${ }^{10}$ method. In all the examples the critical density used was $5 \times 10^{23} \mathrm{~cm}^{-3}$, corresponding to a wavelength of $46.9 \mathrm{~nm} .^{3}$ As this paper concentrates on gain-guided configurations, all the examples presented in this section have maximum density on axis. For density profiles with off-axis maxima, calculations of the output radiation should also include the contribution of the mode of the index waveguide. ${ }^{6,7}$

\section{A. Dependence of the X-Ray Laser Intensity on Plasma-Column Length}

As discussed in the previous section, the length dependence of the output flux is determined by the value of the 
parameter $G_{r}$. To illustrate the influence of the curvature of the density profile, we performed calculations using a set of cubic density profiles that allowed for a continuous tuning of the value of $G_{r}$ while keeping constant the maximum density and radius of the profile. It is important to notice that as the profile is not parabolic, the curvature is not determined by the maximum density and radius, ${ }^{2}$ but is instead an independent and relevant parameter. The set of density profiles used for this example are shown in Fig. 4(a). The curves are labeled by the corresponding value of the parameter $G_{r}$ for $g\left(r_{m}\right)$ $=0.5 \mathrm{~cm}^{-1}$. With the chosen values for the gain, the density profiles in Fig. 4(a) result in the configurations that include values of $G_{r}$ both larger and smaller than 2 , the minimum value required for continued exponential amplification. The gain profile was assumed parabolic and kept the same for all the density profiles in this example. The resulting on-axis output flux after numerical integration is shown in Fig. 4(b) with the different curves also labeled by $G_{r}$. For the highest curvature profiles, with $G_{r}<2$, refraction stops exponential growth. For reduced curvature of the density profiles the value of $G_{r}$ increases and the gain is able to overcome the refraction losses. The effective gain is nevertheless reduced by refraction, and Fig. 4(b) illustrates how the reduction is less important for increasing $G_{r}$ (lower curvature). Further reduction of the curvature $\left(G_{r}>4\right)$ decreases the effect of refraction on the effective gain, but because of the reduced gradients, it also requires a longer plasma column for Eq. (16) to be accurate. For this type of profile a cal-
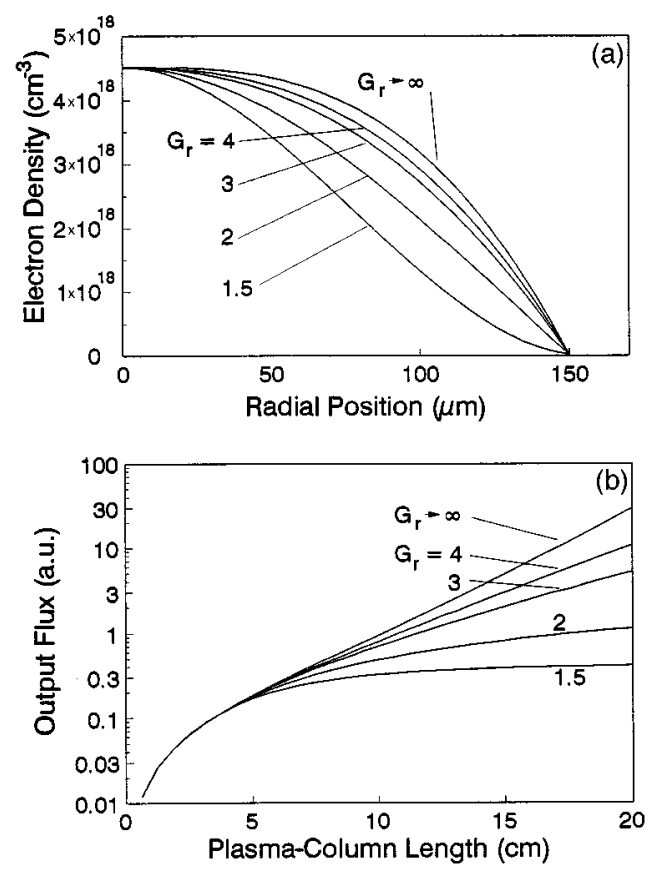

Fig. 4. (a) Electron-density profiles that have a maximum value of $4.5 \times 10^{18} \mathrm{~cm}^{-3}$ on axis, $150-\mu \mathrm{m}$ radius, and $G_{r}$ as labeled for $g_{0}=0.5 \mathrm{~cm}^{-1}$. (b) On-axis output flux computed with the density profiles in (a) and a parabolic gain profile of $150-\mu \mathrm{m}$ radius and $g_{0}=0.5 \mathrm{~cm}^{-1}$.
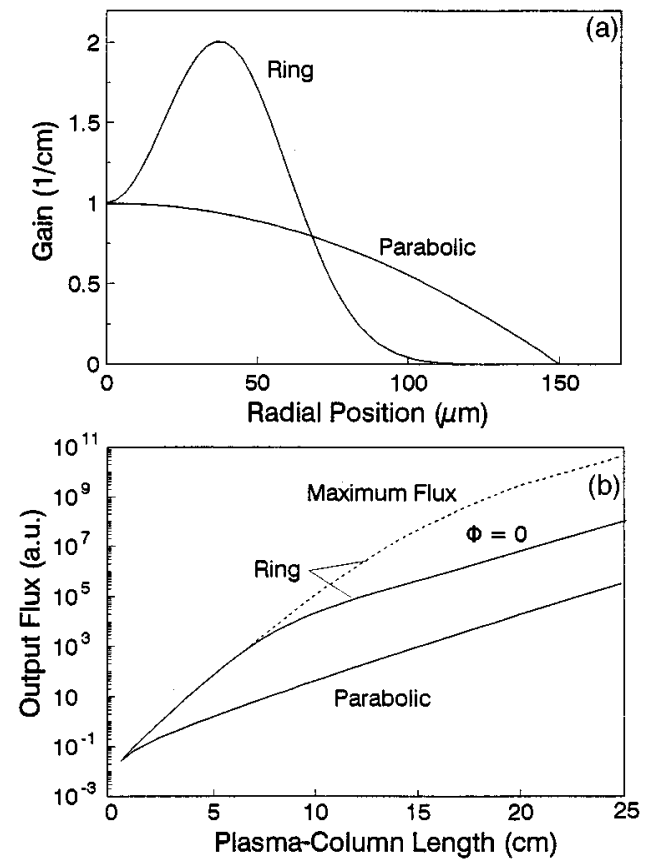

Fig. 5. (a) Gain profiles with maximum on axis (parabolic) and ring geometry (off-axis Gaussian) that have a value $g_{0}$ $=1 \mathrm{~cm}^{-1}$ on axis. (b) Output flux computed with the gain profiles in (a) and a parabolic density profile of $150-\mu \mathrm{m}$ radius and $n_{e}=4.5 \times 10^{18} \mathrm{~cm}^{-3}$. For the parabolic gain profile the maximum flux is on axis; for the ring both the maximum and the on-axis flux are plotted.

culation employing a flat-top index approximation may be more appropriate than the parabolic approximation leading to Eq. (16). This is illustrated by the curves labeled $G_{r} \rightarrow \infty$ in Fig. 4, which show the on-axis output flux for the cubic density profile with $\partial^{2} \eta / \partial r^{2}=0$ on axis. The gain observed in the example (smaller than $g_{0}$ ) is the average gain in the almost-flat region of the density profile, through which nonoptimal rays can travel for the entire length of the plasma column.

Besides its dependence on the curvature of the index of refraction, $G_{r}$ depends on the value of gain at the position of maximum density. The length dependence of the output flux for sufficiently long plasma columns is determined by the value of the gain at just this one point. For plasma columns not long enough for optimal rays to be dominant the whole gain profile contributes to the output flux. As an example, let us consider the gain profiles plotted in Fig. 5 that have the same value of $g_{0}$ $=1 \mathrm{~cm}^{-1}$ on the axis and that are different almost everywhere else. The electron-density profile was chosen parabolic with $150-\mu \mathrm{m}$ radius and $4.5 \times 10^{18} \mathrm{~cm}^{-3}$ maximum density, resulting in $G_{r}=5$ for both profiles. The chosen gain profiles represent different physical situations; the parabolic profile accounts for coincident density and gain maxima, and the off-axis Gaussian profile (ring) accounts for noncoincident gain and density maxima. Figure 5(b) shows the output flux computed with the gain profiles in Fig. 5(a). In this example the ring profile has higher peak gain, and thus its output flux is much larger than that from the parabolic profile. It shows clearly the 
transition to a regime in which the most important contribution comes from optimal rays. The transition is not so evident when the gain and the density maxima coincide on axis. Although the flux from the ring profile grows faster than the parabolic profile for short plasma columns because of the higher maximum gain, after the transition to the regime dominated by optimal rays, the flux from both profiles grows exponentially with an effective gain of $g_{0}\left(1-2 / G_{r}\right)=0.6 \mathrm{~cm}^{-1}$. The plasma column length for which optimal rays become dominant is in reasonable agreement with the crude estimate given in Section 4, which yields a value of $7 \mathrm{~cm}$ for the on-axis emission and roughly twice that length for the off-axis component. For a parabolic profile with $G_{r}>2$ the maximum flux is emitted on axis. For the ring gain profile with maximum density on axis the maximum flux is also emitted on axis for short plasma columns, but longer plasma columns have maximum emission off axis.

\section{B. Beam Patterns}

Figure 6 shows the output beam profile for some of the configurations previously discussed. All the profiles display the narrow peak characteristic of refracted radiation through the sides of the plasma column at $\phi_{r}=3 \mathrm{mrad}$. When refraction dominates and stops the exponential growth, a large proportion of the radiation is bent out and exits the plasma column through the side. It is natural to expect in that case a beam profile with prominent side lobes. This can be seen in Fig. 6 (curve a), which shows the output beam profile for the configuration with $G_{r}$ $=1.5$ in Fig. 4. As discussed in Section 4, when the gain is able to overcome the refraction losses, the output beam pattern consists of two contributions, radiation refracted through the sides of the plasma column and radiation that exits through the end plane of the plasma column. The end-face radiation dominates the beam profiles in Fig. 6 (curves $\mathrm{b}$ and $\mathrm{c}$ ). The configuration in Fig. 4 with $G_{r}=4$, used to obtain curve b in Fig. 6 , has

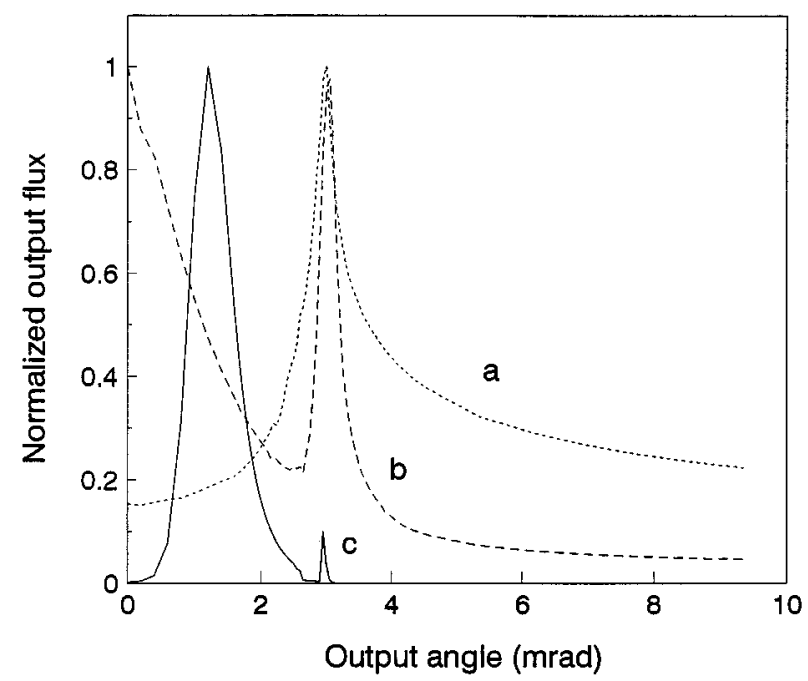

Fig. 6. Variety of output flux profiles computed for a plasma column $20 \mathrm{~cm}$ in length: a, Gain and density profile corresponding to Fig. $4, G_{r}=1.5$. b, Gain and density profile corresponding to Fig. $4, G_{r}=4$. c, Gain and density profile corresponding to the ring gain in Fig. 5 . maximum gain and electron density on axis, and the endface radiation is characterized in that case by a dominant on-axis lobe. For configurations having maximum gain off axis, such as the ring in Fig. 5 used to obtain curve $c$ in Fig. 6, the output beam profile is maximum off axis. The gain and density profiles used in this example produce two separate off-axis maxima, one at $\phi_{r}$ caused by the side radiation and the other caused by the gain profile. For configurations having larger separation of the off-axis gain maxima or steeper density profiles, the off-axis maxima in the output beam profile have larger angular separation. As this angular separation cannot be larger than that of the side radiation peak at $\phi_{r}$, such configurations produce output beam profiles having a single offaxis maximum.

\section{GAIN SATURATION}

For efficient extraction of the energy stored in the laser medium it is desirable to operate in conditions such as to saturate the gain. For the purpose of our discussion we will assume an inhomogeneously broadened transition; analogous results would be obtained for the homogeneous case. The saturation is commonly quantified in the $\mathrm{x}$-ray-laser literature in terms of the gain-length product necessary for saturation of the gain medium. This parameter is highly dependent on the geometry of the plasma column and on the effect of refraction. It is computed $^{11,2}$ by requiring the stimulated-emission rate at the end of the plasma column to be equal to the spontaneous decay rate of the upper laser level. In that case the value of the laser intensity at a point at the end of the plasma columns equals the saturation intensity:

$$
I_{s}=\frac{8 h \nu^{3} \pi}{c^{2} A_{21}\left[\tau_{u}+h_{u} / h_{l}\left(\tau_{l}-A_{21} \tau_{u} \tau_{l}\right)\right]} .
$$

In this equation, $\tau_{u}$ and $\tau_{l}$ are, respectively, the lifetimes of the upper and the lower laser levels. This expression includes the correction for absorption of the lower laser level. ${ }^{12}$ Conversely, the amplified laser intensity is obtained from Eq. (4):

$$
I(l)=S\left\{\exp \left[\int_{0}^{l} g(z) \mathrm{d} z\right]-1\right\} \Delta \Omega .
$$

The influence of the geometry of the plasma column is evident from the presence of the solid-angle factor $\Delta \Omega$. Although the exact calculation of $\Delta \Omega$ requires knowledge of the gain and the density profiles, a good estimate can be obtained when the plasma column can be considered long in the terms discussed in Section 4. By considering only those rays that stay in the plasma column for its entire length and performing the angular integral of the contribution of each ray to the total intensity, we can estimate the solid angle as

$$
\Delta \Omega=\frac{4 \pi\left(d_{g} g_{0}\right)^{2}}{G_{r}{ }^{3}} \exp \left(-2 g_{0} l / G_{r}\right)
$$

with refraction and 


$$
\Delta \Omega=\frac{3 \pi d_{g}^{2}}{g_{0} l^{3}}
$$

when refraction is negligible. In these equations, $d_{g}$ is the FWHM diameter of the gain region, which for simplicity of the calculations was considered parabolic in shape. For different gain profiles the solid angle would differ only by a constant shape factor close to unity. The main effect of refraction is the exponential decrease of the solid angle, responsible for the reduced effective gain. Gainguiding narrowing of the solid angle is especially noticeable in the case without refraction by comparison with the subtended solid angle $\pi d_{g}^{2}\left(4 l^{2}\right)$. For our calculations we will neglect the contribution of the counterpropagating beam.

Owing to saturation, the gain $g$ in Eq. (21) depends on the $z$ position in the plasma column according to

$$
g(z)=\frac{g_{0}}{1+I(z) / I_{s}} .
$$

By replacing Eq. (21) we obtain a differential equation for the gain-length product that can be solved for each particular case. To illustrate the effect of refraction on the saturation process, we solved the resulting differential equation for the particular case of plasma columns of 300 $\mu \mathrm{m}$ in FWHM diameter with $G_{r}=6$ and $G_{r} \rightarrow \infty$ for different values of the peak gain $g_{0}$. The results are shown in Figs. 7 and 8.

In two plasma columns with identical gain profiles but with and without significant refraction, it has been shown that the gain-length $\left(G_{s}=g_{0} l\right)$ necessary for saturation

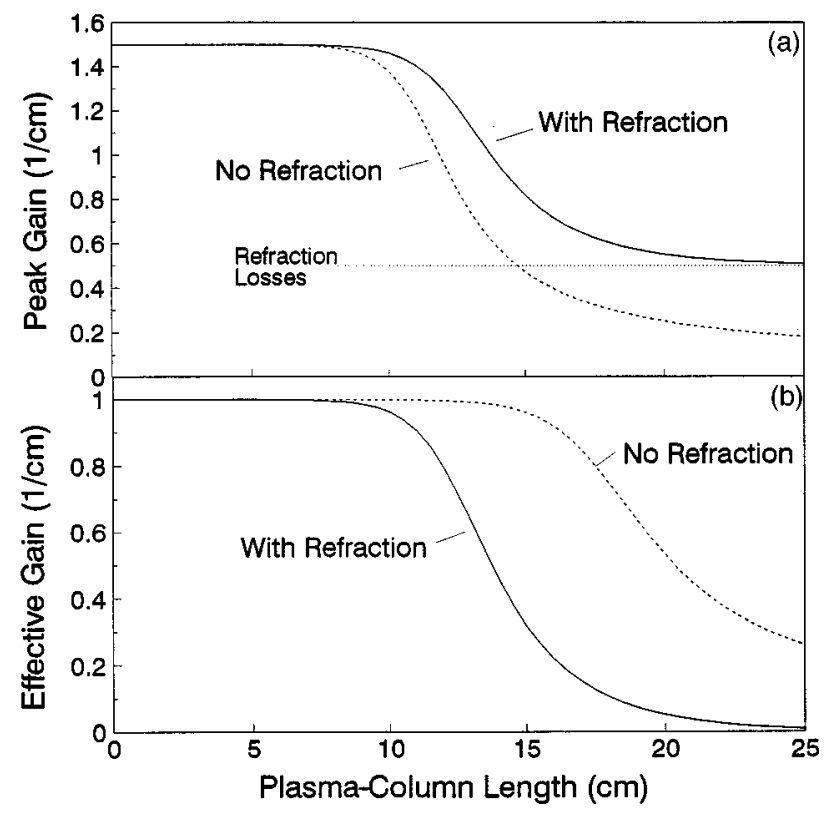

Fig. 7. (a) Dependence of the peak gain $g_{0}$ on the plasmacolumn length for plasma columns with identical gain profiles that have $300-\mu \mathrm{m}$ FWHM diameter and significant $\left(G_{r}=6\right)$ and negligible refraction. The level of refraction losses is indicated. (b) Dependence of the effective gain $g_{\text {eff }}$ on the plasmacolumn length for the refractive plasma column of (a) and a nonrefractive plasma column of the same diameter and effective gain.

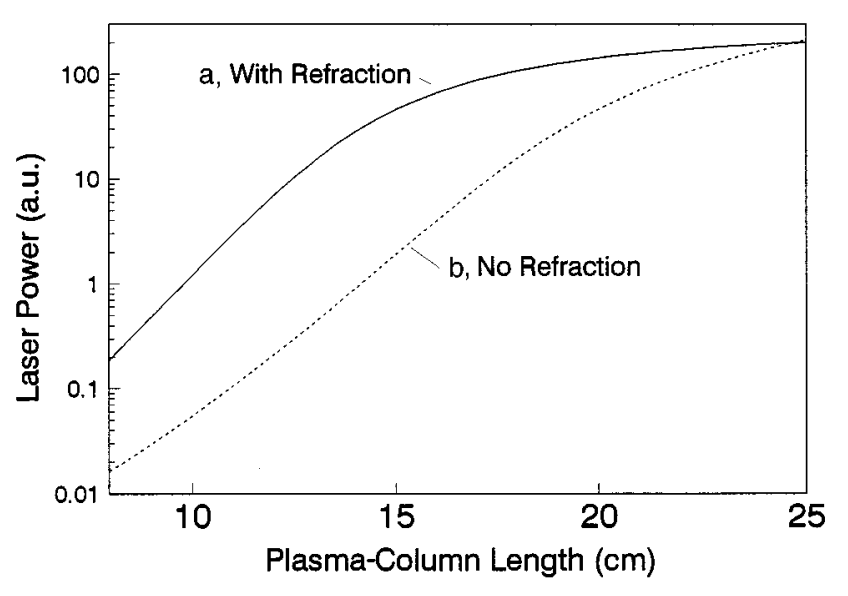

Fig. 8. Length dependence of the laser output power for plasma columns with (curve a) and without (curve b) refraction that have the same effective gain. The parameters employed are the same as in Fig. 7(b).

increases when refraction is important, ${ }^{2}$ as can be expected owing to radiation being bent out of the gain region by refraction. This is shown in Fig. 7 (curve a), where we plot the length dependence of the peak gain for these two cases. However, from the experimental point of view, the gain measured from the slope of the plot of power density versus length is the effective gain $g_{\text {eff }}$ $=g_{0}\left(1-2 / G_{r}\right)$. It is thus useful in analyzing experimental results to compute the saturation gain-length $G_{s}$ in terms of the effective gain, which can be readily obtained from the experimental data, and not in terms of the maximum gain $g_{0}$, which is a priori unknown. When the effect of refraction is assessed by comparing configurations with and without refraction and that have the same effective gain, the effective gain-length necessary for saturation is shorter when refraction is important. In Fig. 7 (curve b) the length dependence of the effective gain obtained by subtracting the refraction losses from the peak gain in Fig. 7 (curve a) is compared with that of a plasma column that has the same effective gain but negligible refraction. The fact that saturation is observed for a shorter plasma-column length when refraction is important can be understood by considering that for equal effective gain the maximum gain, $g_{0}$, must be higher for the refractive case than for the nonrefractive case. The measurable dependence of the laser output power is shown in Fig. 8 for the cases that have the same effective gain. Although the curves in Fig. 8 have the same slope, the refractive case has higher power density because of the fast initial increase at the maximum gain for short plasma columns (see Subsection 4.C). Another important consequence of refraction can be noticed from the plots in Fig. 7. In contrast with the saturation behavior without refraction, after saturation occurs, the gain rapidly reduces to a value slightly over that necessary to compensate for refraction losses. The measurable effect is a more pronounced reduction of the amplification after saturation than that predicted without taking refraction into account, in good agreement with experimental observations (see Refs. 13 and 14 and Fig. 9). 


\section{ANALYSIS OF CAPILLARY DISCHARGE SOFT-X-RAY LASER EXPERIMENTS}

In this section we use the theoretical tools developed in this paper to analyze experimental results of amplification at $46.9 \mathrm{~nm}$ in Ne-like argon in the plasma column of a fast compressional capillary discharge. The laser pulse characteristics, the dynamics of the capillary plasma column, and the experimental setup utilized have been discussed in previous publications. ${ }^{3,4,13}$ The measurements discussed below were conducted in polyacetal capillaries 4 $\mathrm{mm}$ in diameter filled with $720 \mathrm{mTorr}$ of pure argon gas, excited by current pulses of $(39 \pm 1.5) \mathrm{kA}$ peak current having a first half cycle duration of $(75 \pm 3) \mathrm{ns}$.

The measured laser output power shown in Fig. 9(a) grows exponentially over several orders of magnitude before saturating. The measured beam divergence shown in Fig. 9(b) rapidly decreases for plasma columns up to 10 $\mathrm{cm}$ and stays approximately constant for longer columns, indicating that the experiments are in the long plasmacolumn regime. For capillaries $10 \mathrm{~cm}$ and longer the beam characteristics should be therefore very similar to the mode of the gain-guided waveguide. Far-field beam profiles characterized by a single central peak were obtained in some experiments, while prominent side lobes were observed in other instances. Profiles with side lobes cannot be accounted for without the consideration of refraction. The observed angular separation of the side lobes is indicative of an electron density of 4

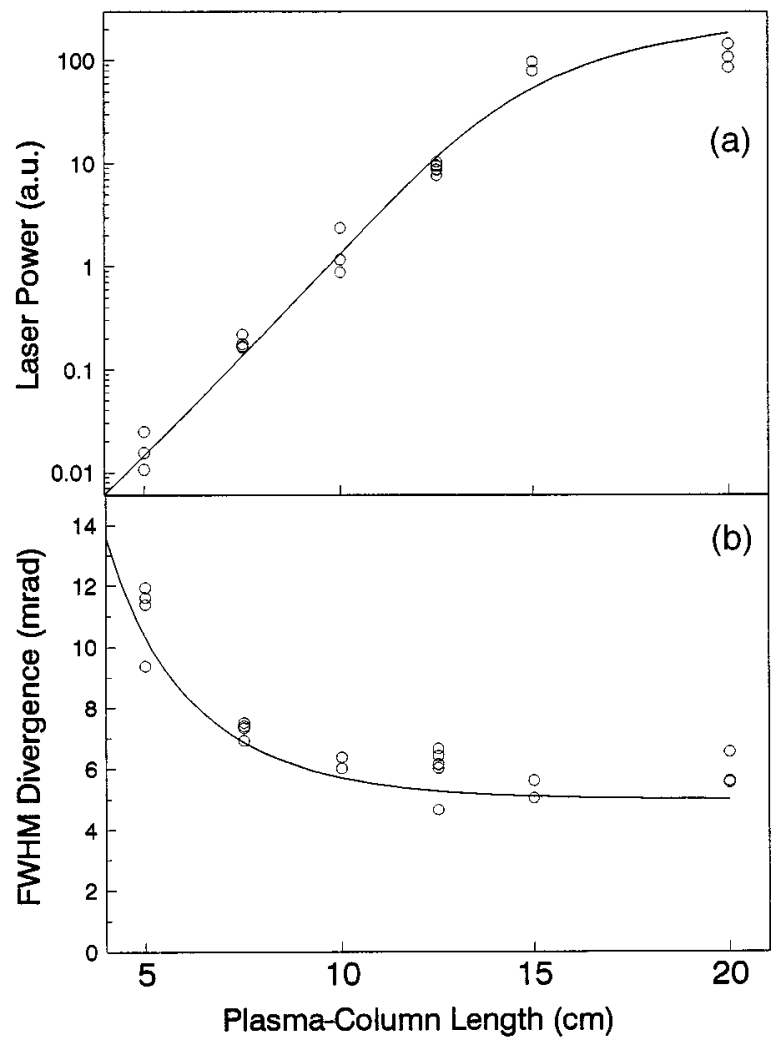

Fig. 9. Measured (data points) and computed (solid curves) dependence of (a) the output laser power and (b) the laser beam divergence of the 46.9-nm line of Ne-like Ar with capillary plasmacolumn length. The theoretical curves correspond to $G_{r}=6$, $g_{0}=1.48 \mathrm{~cm}^{-1}$, and $d_{g}=290 \mu \mathrm{m}$. $\times 10^{18} \mathrm{~cm}^{-3}$. Regardless of the observed profile, the value of the effective gain was similar, and ranged from 0.85 to $1.16 \mathrm{~cm}^{-1}$ in several different series of measurements.

As discussed in previous sections, when the soft-x-ray laser is in the long plasma-column regime, the refraction gain-length $G_{r}$ and the spatial extent of the gain region determine the amplification and the saturation behavior and provide an estimate for the beam divergence. The solid curves in Fig. 9 were obtained from calculations conducted by the long plasma approximation and by adjusting $g_{0}, G_{r}$, and $d_{g}$ to reproduce the measured amplification, saturation, and beam divergence. The resulting values of the parameters, $g_{0}=1.48 \mathrm{~cm}^{-1}, G_{r}$ $=5.9$, and $d_{g}=290 \mu \mathrm{m}$, are in reasonable agreement with those obtained from hydrodynamic/atomic physics calculations. $^{15,16}$ For the range of densities predicted by these calculations $\left(3-10 \times 10^{18} \mathrm{~cm}^{-3}\right)$ the obtained value of $G_{r}$ corresponds to density profiles slightly more peaked than parabolic, such as those shown in Fig. 4(a). The theoretical curves reproduce well the features observed in the experiment for both the laser power and the beam divergence.

The computations show that, in this series of measurements, saturation of the gain was achieved, a conclusion supported by recent measurements of the laser pulse output energy. ${ }^{16}$ The saturation intensity is calculated to be reached at plasma-column lengths of $\sim 16 \mathrm{~cm}$ when the gain-length product is 15.5. The saturation power density (56 MW/ $\mathrm{cm}^{2}$ ) was computed assuming an ion temperature of $100 \mathrm{eV}$ and an effective- to radiative-lifetime ratio for the laser upper level of 20 , as indicated by computations for plasma densities of $5 \times 10^{18} \mathrm{~cm}^{-3} \cdot 15$ Other series of measurements in which slightly larger effective gain coefficients (up to $1.16 \mathrm{~cm}^{-1}$ ) were obtained have shown saturation at correspondingly shorter plasma-column lengths, as can be expected from gain saturation. In Fig. 7(b) it is observed that the divergence measured on 20-cm-long capillaries is slightly larger than that for capillaries $15 \mathrm{~cm}$ in length. This is consistent with the rebroadening that is to be expected when the effect of gain guiding in compensating refraction is reduced by gain saturation. As the calculation of the beam divergence in Fig. 9(b) did not include saturation, the theoretical curve does not show this rebroadening.

\section{CONCLUSION}

We have analyzed soft-x-ray beam propagation and amplification in gain-guided amplifiers of cylindrical geometry for the general case of arbitrary gain and density profiles. Two distinct regimes in the length dependence of the output intensity are identified, one for short plasma columns in which all rays emitted within the solid angle of the amplifier contribute significantly to the output beam, and the other for long plasma columns, which is dominated by the gain experienced by the particular set of optimal rays. We obtained an estimate of the plasmacolumn length for which optimal rays become dominant. For sufficiently long plasma columns the length dependence of the intensity is determined solely by the gain and the curvature of the density profile at the position of 
maximum density. This dependence is characterized by the refraction gain-length $G_{r}$ introduced in connection with parabolic profiles ${ }^{2}$ for which we obtained a general expression valid for arbitrary profiles. A general condition to be fulfilled by the gain and the density profiles for continued exponential growth up to the onset of saturation $\left(G_{r}>2\right)$ was obtained. Refraction is shown to reduce the gain coefficient by a factor $1-2 / G_{r}$. For long plasma columns the output beam pattern consists of two contributions, one produced by radiation exiting through the end plane of the plasma column, which has a shape determined by the gain and density profiles, and the other a narrow ring produced by the radiation bent out of the plasma column by refraction. The side radiation is centered at an angle $\phi_{r}$, which, as in the unidimensional case ${ }^{2}$ depends only on the value of the maximum electron density. We analyzed the case of amplifiers with gainlength approaching saturation by considering that the gain measured in an experiment is the effective gain, $g_{\text {eff }}=g_{0}\left(1-2 / G_{r}\right)$, as reduced by refraction. It is shown that the saturation gain-length computed by the measured effective gain without taking refraction into account overestimates the gain-length necessary for gain saturation. The theoretical analysis developed in this paper was employed to analyze the results of a capillary discharge soft-x-ray laser experiment that displays the effects of refraction and gain saturation.

\section{ACKNOWLEDGMENTS}

We thank V. Shlyaptsev for useful discussions. This work was supported by National Science Foundation grant ECS-9401952 and the Colorado Advanced Technology Institute.

\section{REFERENCES}

1. R. C. Elton, X-Ray Lasers (Academic, Boston, Mass., 1990).

2. R. A. London, "Beam optics of exploding foil plasma x-ray lasers," Phys. Fluids 31, 184-192 (1988).
3. J. J. Rocca, V. Shyaptsev, F. G. Tomasel, O. D. Cortazar, D. Hartshorn, and J. L. A. Chilla, "Demonstration of a discharge pumped table-top soft x-ray laser," Phys. Rev. Lett. 73, 2192-2195 (1994).

4. J. J. Rocca, F. G. Tomasel, M. C. Marconi, V. N. Shlyaptsev, J. L. A. Chilla, B. T. Szapiro, and G. Giudice, "Dischargepumped soft x-ray laser in neon-like argon," Phys. Plasmas 2, 2547-2554 (1995).

5. E. E. Fill, "Gain guiding of x-ray laser beams," Opt. Commun. 67, 441-445 (1988).

6. C. G. DurfeeIII, J. Lynch, and H. M. Milchberg, "Mode properties of a plasma waveguide for intense laser pulses," Opt. Lett. 19, 1937-1939 (1994).

7. H. M. Milchberg, C. G. Durfee III, and J. Lynch, "Application of a plasma waveguide to soft-x-ray lasers," J. Opt. Soc. Am. B 12, 731-737 (1995).

8. M. Born and E. Wolf, Principles of Optics, 2nd ed. (Pergamon, Oxford, 1964).

9. T. P. Hughes, Plasmas and Laser Light (Wiley, New York, 1975).

10. B. Carnahan, H. A. Luther, and J. O. Wilkes, Applied Numerical Methods (Wiley, New York, 1969).

11. A. E. Siegman, Lasers (University Science, Mill Valley, Calif., 1986).

12. J. T. Verdeyen, Laser Electronics, 2nd ed. (Prentice-Hall, Englewood Cliffs, N.J., 1989).

13. J. J. Rocca, M. C. Marconi, J. L. A. Chilla, D. P. Clark, F. G. Tomasel, and V. N. Shlyaptsev, "Discharge-driven 46.9-nm amplifier with gain-length approaching saturation," IEEE J. Sel. Topics Quantum Electron. 1, 945-948 (1995).

14. A. Carillon, H. Z. Chen, P. Dhez, L. Dwivedi, J. Jacoby, P. Jaegle, G. Jamelot, J. Zhang, M. H. Key, A. Kidd, A. Klishnick, R. Kodama, J. Krishnan, C. L. S. Lewis, D. Neely, P. Norreys, D. O’Neill, G. J. Pert, S. A. Ramsden, J. P. Raucourt, G. J. Tallents, and J. Uhomoibhi, "Saturated and near-diffraction limited operation of an XUV laser at 23.6 nm," Phys. Rev. Lett. 68, 2917-2920 (1992).

15. V. N. Shlyaptsev, J. J. Rocca, and A. L. Osterheld, "Dynamics of a capillary discharge x-ray laser," Proc. SPIE 2520, 365-371 (1995).

16. J. J. Rocca, D. P. Clark, J. L. A. Chilla, and V. N. Shlyaptsev, "Energy extraction and achievement of the saturation, limit in a discharge pumped table-top soft x-ray amplifier," Phys. Rev. Lett. 77, 1476-1479 (1996). 